\title{
Volatile anesthetic sevoflurane ameliorates endotoxin-induced acute lung injury via microRNA modulation in rats
}

\author{
TATSURO OTSUKI, MASASHI ISHIKAWA, YOKO HORI, GENTARO GOTO and ATSUHIRO SAKAMOTO \\ Department of Anaesthesiology and Pain Medicine Graduate School of Medicine, \\ Nippon Medical School, Bunkyo-ku, Tokyo 113-8603, Japan
}

Received December 11, 2014; Accepted January 29, 2015

DOI: $10.3892 /$ br.2015.428

\begin{abstract}
Volatile anesthetics have a lung protective effect in acute lung injury (ALI). Our previous study showed sevoflurane affects the expression of microRNA (miRNA) that control various physiological systems by regulating messenger RNA (mRNA) expression. However, the association between the anti-inflammatory effect of sevoflurane and miRNAs modulation remains unknown. The aim of the present study was to investigate the effect of sevoflurane and the expression of miRNAs in an endotoxin-induced ALI model in rats. Wistar rats were randomly assigned to three groups [lipopolysaccharide (LPS), LPS-sevoflurane and control; $n=8 /$ group]. All the rats were mechanically ventilated and intravenously-administered LPS (saline as control). Two hours post-injury, general anaesthesia was performed for $4 \mathrm{~h}$ with $2 \%$ sevoflurane (LPS-sevoflurane). The LPS and the control groups did not receive anaesthesia. The severity of ALI was evaluated by partial pressure of oxygen/fraction of inspired oxygen and the mRNA expression of inflammatory cytokine. The miRNA expression in lung tissue was analyzed by a reverse transcription-quantitative polymerase chain reaction. LPS caused ALI, evidenced by the impairment of pulmonary function and increased mRNA levels of tumor necrosis factor- $\alpha$, interleukin- 6 and nuclear factor- $\kappa \mathrm{B}$. Sevoflurane improved pulmonary function and inhibited the increased mRNAs. Of the 219 miRNAs detected, 15 and nine miRNAs were significantly changed in the LPS and LPS-sevoflurane group, respectively. In the LPS-sevoflurane group, the expression of several miRNAs that regulate inflammation was significantly changed compared to the LPS group. In conclusion, the present data showed that sevoflurane influences the expression of the miRNAs that regulate
\end{abstract}

Correspondence to: Mr. Tatsuro Otsuki, Department of Anaesthesiology and Pain Medicine Graduate School of Medicine, Nippon Medical School, 1-1-5 Sendagi, Bunkyo-ku, Tokyo 113-8603, Japan

E-mail: truant7140@nms.ac.jp

Key words: volatile anaesthetics, sevoflurane, microRNA, acute lung injury, postconditioning inflammation. This result suggests that the changes in miRNA expression are involved in the lung protective mechanisms of volatile anesthetics.

\section{Introduction}

Acute lung injury (ALI) is a major complication in critically ill patients (1). Therefore, it is important to preserve lung function and prevent further exacerbation for those patients whose lungs are already vulnerable prior to surgery. The volatile anesthetic sevoflurane is known to have organ protective effects in several pathological conditions. For example, Voigtsberger et al (2) have recently shown that sevoflurane ameliorates gas exchange and attenuates lung damage in a model of lipopolysaccharide (LPS)-induced ALI.

MicroRNA (miRNA) is a newly discovered and non-coding RNA that regulates genome expression at the post-transcriptional level (3). miRNA is estimated to control $30-90 \%$ of human genes (4). miRNA has been suggested to either suppress messenger RNA (mRNA) translation or reduce mRNA stability by binding particular regions of mRNA (5). Recent published data have also revealed that the expression and function of miRNA are associated with a broad range of diseases.

In our previous study, it was found that sevoflurane affects the expression of miRNAs in normal rat lung (6). However, such effects of anesthetic ALI remain unknown. Therefore, we hypothesized that sevoflurane affects the miRNAs that regulate inflammatory responses and investigated changes of miRNA in an LPS-induced ALI model in rats.

\section{Materials and methods}

Approval. The study was approved by the Animal Research Committee of Nippon Medical School, Tokyo, Japan (approval no. 22-147).

Sample preparation. Male Wistar rats (Tokyo Experimental

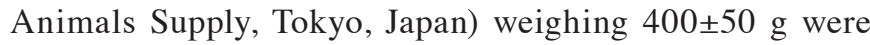
housed at $22 \pm 1^{\circ} \mathrm{C}$ under a 12 -h light/12-h dark cycle. The rats freely accessed food ad libitum and water. All the rats were anesthetized by intra-peritoneal injection of sodium pentobarbital $(50 \mathrm{mg} / \mathrm{kg})$ for the surgical procedure and subsequently maintained with hourly injections $\left(15 \mathrm{mg} \mathrm{kg}^{-1}\right)$ when 
necessary. The rats were placed supine on a heating blanket under a heating lamp to maintain a body temperature of $37^{\circ} \mathrm{C}$ throughout the experiments. For continuous anesthetic infusion and fluid administration, the tail vein was cannulated with a sterile 24-gauge catheter and normal saline was administered via the catheter at a rate of $5 \mathrm{ml} \mathrm{kg}^{-1} \mathrm{~h}^{-1}$. A sterile 24-gauge polyethylene catheter was placed in the left femoral artery for blood sampling and blood pressure monitoring. All the rats were tracheotomized and a sterile 14-gauge catheter was inserted into the trachea, followed by mechanical ventilation (rodent ventilator model 638; Harvard Apparatus, Holliston, MA, USA) in the volume-controlled mode $\left(8 \mathrm{ml} \mathrm{kg}^{-1}\right.$ tidal volume) without positive end-expiratory pressure. The fraction of inspired oxygen $\left(\mathrm{FiO}_{2}\right)$ concentration was 1.0. The inspiratory-expiratory ratio was 1:2 and respiratory frequency was 60-70 $\mathrm{min}^{-1}$.

The rats were randomly assigned to three different groups (n=8/group): i) Control, ii) LPS and iii) LPS-sevoflurane. Rats in the control and LPS groups were intravenously injected with $10 \mathrm{mg} \mathrm{kg}^{-1}$ Escherichia coli-LPS (serotype 055:B5; Sigma Aldrich, St. Louis, MO, USA) dissolved in $1 \mathrm{ml}$ of normal saline. The control group received an equal volume of normal saline. The injection was defined as 0 time point. Two hours after the injection, the LPS-sevoflurane group received 2.0\% sevoflurane (Maruishi Pharmaceutical, Co., Ltd., Osaka, Japan) for $4 \mathrm{~h}$, but not the control and LPS group. At the end of the $6 \mathrm{~h}$ experimental period, arterial blood gas and mean arterial pressure (MAP) were measured and the arterial oxygen tension to inspired oxygen fraction [partial pressure of oxygen $\left.\left(\mathrm{PaO}_{2}\right) / \mathrm{FiO}_{2}\right]$ was calculated. Finally, all the rats were sacrificed by decapitation. The right lungs were obtained immediately and washed with phosphate-buffered saline and stored in RNAlater ${ }^{\circledR}$ (Life Technologies, Applied Biosystems, Carlsbad, CA, USA) at $-80^{\circ} \mathrm{C}$ until use.

Quantification of miRNA and $m R N A$ expression by reverse transcription-quantitative polymerase chain reaction $(R T-q P C R)$. RT-qPCR was performed as described previously (7). Total RNA was isolated from the lung samples using a mirVana miRNA isolation $\mathrm{kit}^{\circledR}$ (Ambion, Life Technologies, Waltham, MA, USA) according to the manufacturer's instructions. RNA amounts and purity were assessed by absorbance at $260 \mathrm{~nm}$ and 260/280-nm ratio, respectively, with a NanoDrop ND-1000 (Thermo Fisher Scientific Inc., Waltham, MA, USA). Samples with an A260/280-nm of $\geq 1.8$ were qualified for qPCR.

Quantification of mature miRNA expression was performed using the TaqMan microRNA reverse transcription kit, TaqMan Universal PCR Mastermix, Megaplex Reverse Transcription primer Pool A/B and TLDA Rodent MicroRNA card A/B (Life Technologies, Applied Biosystems). Each card contains 373 preloaded rat miRNA targets, all catalogued in the miRNA database. Mann U6 was selected as an endogenous control. All procedures were performed according to manufacturer's protocols. Amplification and detection were performed using a 7900 HT real time PCR System (Life Technologies, Applied Biosystems). For quantification of miRNA expression, the comparative $\mathrm{Ct}$ method $(\Delta \Delta \mathrm{Ct})$ was used. The target gene was quantified relative to the control gene and is expressed as fold change, calculated as $2^{-\Delta \Delta \mathrm{Ct}}$.
Table I. Effects of $6 \mathrm{~h}$ anesthetic treatment on LPS-induced alterations of $\mathrm{PaO}_{2}, \mathrm{PaCO}_{2}$ and $\mathrm{MAP}$.

\begin{tabular}{lccc}
\hline Variables & Control & LPS & LPS-sevo \\
\hline $\mathrm{PaO}_{2}$ & $544 \pm 34$ & $293 \pm 24^{\mathrm{a}}$ & $375 \pm 26^{\mathrm{a}, \mathrm{b}}$ \\
$\mathrm{PaCO}_{2}$ & $35.7 \pm 4.0$ & $55.3 \pm 6.1^{\mathrm{a}}$ & $45.2 \pm 4.5^{\mathrm{a}, \mathrm{b}}$ \\
$\mathrm{MAP}$ & $126 \pm 5$ & $96 \pm 5$ & $93 \pm 7$ \\
\hline
\end{tabular}

Data are mean \pm standard deviation in $\mathrm{mmHg}$. Partial arterial oxygen tension $\left(\mathrm{PaO}_{2}\right)$, partial arterial carbon dioxide tension $\left(\mathrm{PaCO}_{2}\right)$ and mean arterial pressure (MAP) were determined in the four study groups at $6 \mathrm{~h}$. Fraction of inspired oxygen $\left(\mathrm{FiO}_{2}\right)=1.0$. ${ }^{\mathrm{a}} \mathrm{P}<0.05$ vs. control, ${ }^{\mathrm{b}} \mathrm{P}<0.05$ vs. lipopolysaccharide (LPS). Sevo, sevoflurane.

For mRNA analysis, a High Capacity cDNA RT kit (Life Technologies, Applied Biosystems) and TaqMan mRNA assay primers for tumor necrosis factor- $\alpha$ (TNF- $\alpha$, cat. no. 4331182), interleukin-6 (IL-6 cat. no. 4331182) and nuclear factor- $\kappa \mathrm{B}$ $(N F-\kappa B$, cat. no. 4351372) were used with $1 \mathrm{ng}$ of total RNA/reaction. Reverse transcription was performed at $20^{\circ} \mathrm{C}$ for $5 \mathrm{~min}, 42^{\circ} \mathrm{C}$ for $30 \mathrm{~min}$ and $95^{\circ} \mathrm{C}$ for $5 \mathrm{~min}$ on a GeneAmp ${ }^{\circledR}$ 5700 system (Life Technologies, Applied Biosystems). Quantification of mRNA expression was performed in the same manner following normalization to GAPDH and is expressed relative to the control group. The data analysis was performed using DataAssist software v2.0 (Life Technologies, Applied Biosystems).

Statistical analysis. All the data are expressed as means \pm standard deviation. For multiple group comparisons, analysis of variance followed by Tukey's test $(\mathrm{P}<0.05)$ was performed to compare the physiological data and the relative expression of mRNA. Tukey's test $(\mathrm{P}<0.01)$ was applied to identify miRNAs that were differentially expressed compared to the control group. Statistical analysis was performed using GraphPad Prism 5 (GraphPad Software Inc., San Diego, CA, USA).

\section{Results}

Physiological data. Arterial blood gas analysis showed that the rats administered LPS had a significant decrease in $\mathrm{PaO}_{2} / \mathrm{FiO}_{2}\left(\mathrm{FiO}_{2}=1.0\right)$ compared to the control $6 \mathrm{~h}$ post-injury. Among the two LPS groups, however, the LPS-sevoflurane group had significantly higher $\mathrm{PaO}_{2} / \mathrm{FiO}_{2}$ and lower $\mathrm{PaCO}_{2}$ compared to the LPS groups (Table I). The MAP in the two LPS groups was significantly lower than that of the control. There were no significant differences in MAP among the two LPS groups.

Expression of miRNA. RT-qPCR analysis showed that 219 miRNAs out of 373 miRNAs examined were expressed. Relative expression ratios of miRNAs compared to the control group are presented in Table II. There were 23 differentially expressed miRNAs in the LPS group. In the LPS group, miR-155 and miR-146a showed marked increases; $19.36 \pm 4.47$ and $7.32 \pm 2.01$, respectively. Following sevoflurane anesthesia, $m i R-155$ was significantly decreased $(8.01 \pm 2.23)$ but $m i R-146$ had no significant change (8.67 \pm 2.27$)$ from LPS alone (Table II). 
Table II. LPS-responsive miRNAs in lung tissue.

\begin{tabular}{|c|c|c|c|c|}
\hline \multirow[b]{2}{*}{ Assay } & \multicolumn{2}{|c|}{ LPS } & \multicolumn{2}{|c|}{ LPS-sevo } \\
\hline & Fold change & $\mathrm{P}$-value & Fold change & P-value \\
\hline let $-7 a$ & $0.31 \pm 0.22$ & 0.002 & $0.38 \pm 0.30$ & 0.007 \\
\hline let-7c & $0.51 \pm 0.16$ & 0.008 & $0.48 \pm 0.29$ & 0.005 \\
\hline let-7d & $0.50 \pm 0.20$ & 0.002 & $0.57 \pm 0.17$ & 0.003 \\
\hline let-7i & $0.33 \pm 0.15$ & $<0.008$ & $0.49 \pm 0.27^{\mathrm{a}}$ & 0.01 \\
\hline$m i R-9$ & $2.21 \pm 0.91$ & 0.009 & $2.18 \pm 0.82$ & 0.006 \\
\hline$m i R-125 b$ & $0.27 \pm 0.13$ & 0.004 & $0.33 \pm 0.14$ & 0.001 \\
\hline$m i R-127$ & $3.81 \pm 2.04$ & 0.001 & $3.49 \pm 1.27$ & 0.001 \\
\hline$m i R-146 a$ & $7.32 \pm 2.01$ & $<0.01$ & $8.67 \pm 2.27$ & $<0.001$ \\
\hline$m i R-155$ & $19.36 \pm 4.47$ & $<0.001$ & $8.01 \pm 2.23^{\mathrm{a}}$ & $<0.001$ \\
\hline$m i R-301 a$ & $0.29 \pm 0.20$ & 0.004 & $0.38 \pm 0.24$ & 0.006 \\
\hline$m i R-301 b$ & $0.28 \pm 0.26$ & 0.005 & $0.36 \pm 0.30$ & 0.006 \\
\hline$m i R-30 e$ & $1.58 \pm 0.32$ & 0.009 & $1.82 \pm 0.48$ & 0.008 \\
\hline$m i R-322$ & $0.58 \pm 0.14$ & 0.003 & $0.45 \pm 0.27$ & 0.006 \\
\hline$m i R-340-5 p$ & $2.96 \pm 0.61$ & $<0.001$ & $3.26 \pm 1.18$ & 0.002 \\
\hline$m i R-425$ & $2.03 \pm 0.36$ & 0.006 & $1.88 \pm 0.28$ & 0.007 \\
\hline
\end{tabular}

miRNAs that were significantly increased or decreased compared to the control $(\mathrm{P}<0.01)$. Values are the mean fold change \pm standard deviation in each group. P-value, statistical results of Tukey's test. ${ }^{\mathrm{a}} \mathrm{P}<0.01$ vs. lipopolysaccharide (LPS). Sevo, sevoflurane.

A

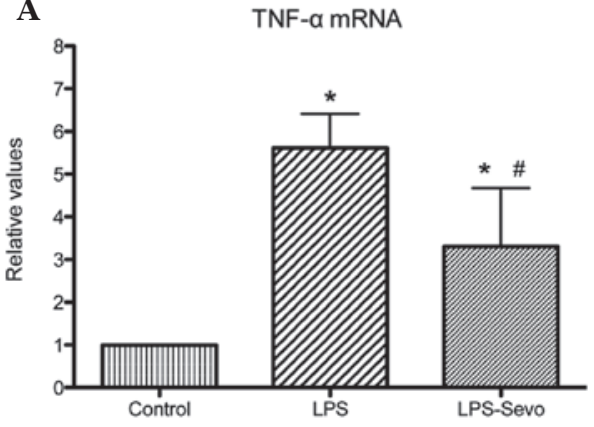

B

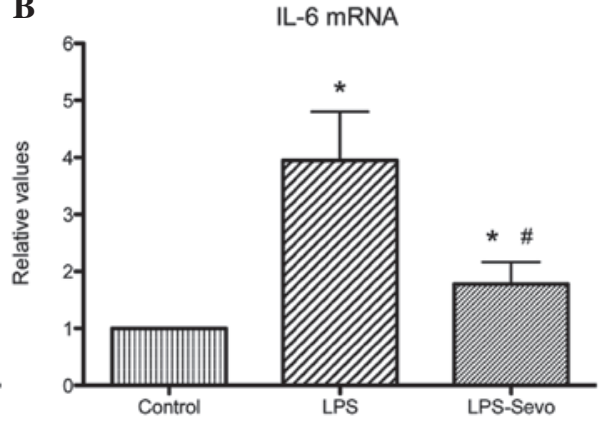

C

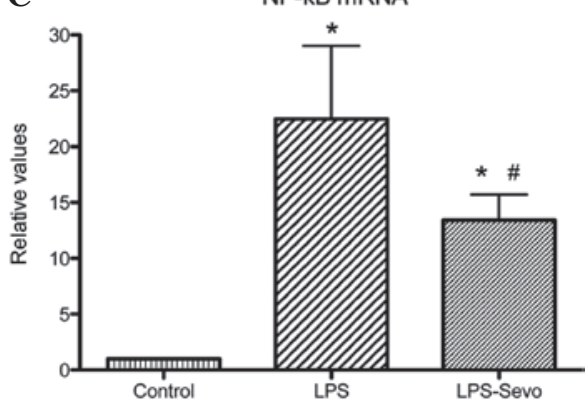

Figure 1. Anesthetics ameliorate lipopolysaccharide (LPS)-induced increases in inflammatory cytokine gene expression in lung. Evaluation of (A) tumor necrosis factor- $\alpha(T N F-\alpha),(\mathrm{B})$ interleukin-6 (IL-6) and $(\mathrm{C})$ nuclear factor- $\kappa \mathrm{B}(N F-\kappa B)$ messenger RNA expression in lung tissue. Data are expressed relative to the normalized mRNA level in the control group. Data are mean \pm standard deviation. ${ }^{*} \mathrm{P}<0.05$ vs. control. ${ }^{*} \mathrm{P}<0.05 \mathrm{vs}$. LPS.

Expression of $m R N A$. Compared to the control group, mRNA expression of $T N F-\alpha, I L-6$ and $N F-\kappa B$ were significantly increased in the two groups treated with LPS. Compared to the LPS group, the expression of $T N F-\alpha, I L-6$ and $N F-\kappa B$ mRNA decreased significantly in the LPS-sevoflurane group (Fig. 1).

\section{Discussion}

The main finding of the present study was that sevoflurane anesthesia not only improves oxygenation and ameliorates inflammation, but also alters miRNA expression in an ALI rat model. Notably, some of the significantly expressed miRNAs in the study are known to regulate the innate immune system against inflammation induced by LPS per se. This system is triggered by the recognition of LPS by Toll-like receptor 4 (TLR4) and the signal activates intracellular signaling cascades that result in $\mathrm{NF}-\kappa \mathrm{B}$ activation and subsequent production of inflammatory cytokines, including TNF- $\alpha$ and IL-6 (8). Recent studies in macrophages, neutrophils, monocytes, epithelial cells and even whole lungs have shown that activation of TLR 4 and TNF- $\alpha$ receptors results in rapid expression of miRNAs, including let-7, miR-9, miR-99b, miR-125a/b, miR-132, miR-146a, miR-155, miR-187 and miR-223 (9-12). Of these, let-7, miR-9, miR-125a, miR-146a and $m i R-155$ were significantly upregulated by LPS as demonstrated in the study.

The present data showed that the expression of $m i R-155$ is enhanced by LPS stimulation and sevoflurane had an ameliorating effect. This change parallels with the changes in the expression of inflammatory cytokine mRNAs. Tili et al (13) showed that mice overexpressing $m i R-155$ in B-cells produced more $T N F$ - $\alpha$ when challenged with LPS and the increase of $T N F-\alpha$ accelerated the onset of mortality. This finding established that the upregulation of $m i R-155$ in response to LPS is 
an indicator of an increased sensitivity to endotoxin stimulation. Collectively, these results suggest that volatile anesthetics attenuate the toxic effect of LPS by affecting $m i R-155$ expression. This finding highlights the importance of further investigation into whether blocking $m i R-155$ can be used as therapeutic intervention to dampen excessive inflammation.

Several studies have indicated that $m i R-146 a$ negatively regulates the activation of inflammatory pathways by controlling the TLR4 pathway through a negative-feedback mechanism (9). During the inflammatory response to microbial invasion, $m i R-146 a$ is shown to increase continuously and remain at a high level, exerting negative feedback effects on several mRNAs of adaptor kinases downstream of TLR4 signal transduction. In this upregulated $m i R-146 a$ state, monocytes and neutrophils no longer produce inflammatory mediators. This phenomenon is referred to as endotoxin-tolerance (14). In the present study, the LPS challenge enhanced miR-146a expression and this result is consistent with previous studies. However, administration of sevoflurane did not alter the expression of $m i R-146 a$ significantly. A possible explanation of this result is that $m i R-146 a$ may not be involved in the lung protective mechanisms of sevoflurane. Another explanation is that the statistical differences were not reached due to 'mixture' sample of whole lung homogenates, which include monocytes, neutrophils, macrophages, epithelial cells and blood vessels. This means that the miRNA expression profile reflects the sum of these different cells. For this reason, significant differences between groups could not be detected. To clarify the role of miR-146a in the immunomodulatory effect of anesthetic agents, it is important to determine the expression of $m i R-146 a$ in the various cell types participating in inflammation.

Current knowledge of the organ protective effects of anesthetic agents mainly originates from studies of ischemia-reperfusion injury $(15,16)$. The administration of a volatile anesthetic prior toischemia, known as preconditioning, has been shown to attenuate ischemia-reperfusion-induced injury in the heart, lung, kidney and liver (17-19). The preconditioning effects of volatile anesthetics were also confirmed in an endotoxin-induced ALI model (20). Although preconditioning appears to be an effective approach, the clinical applicability of this phenomenon is limited, as treatments are usually initiated following the onset of a trigger event. By contrast, postconditioning would be more applicable in a clinical situation. Hofstetter et al (21) examined the postconditioning effect of sevoflurane in an in vivo model of LPS-induced endotoxemia in rats, where administration of sevoflurane $15 \mathrm{~min}$ after intravenous injection of LPS resulted in decreased TNF- $\alpha$ and IL- $1 \beta$. The present study demonstrated that postconditioning is effective even $2 \mathrm{~h}$ after LPS stimulation and indicates anesthetic postconditioning could be initiated in a later phase of clinical treatment. However, these lung protective effects were confirmed only during anesthesia and changes of lung function and inflammation following anesthesia remain unknown. Further investigations of temporal changes of miRNA, inflammatory cytokine expression and mortality following anesthesia are required.

The present study has certain limitations. First, individual miRNAs bind to multiple target mRNAs and mRNAs are not regulated by a single miRNA. Further research is required to identify the specific associations between each miRNA and mRNA using antagonists or transgenic animal models. Second, a limited number of mediators associated with the innate immune system by TLR4 were examined. There are a number of other constitutive mediators in the pathways and the association between these mediators and miRNAs requires further investigation.

In conclusion, the present study revealed that the miRNA expression patterns are altered by sevoflurane anesthesia in a rat LPS-induced ALI model. These results suggested that the expression and function of miRNA are involved in the fundamental mechanism of the lung protective effect of volatile anesthetics.

\section{References}

1. Rubenfeld GD, Caldwell E, Peabody E, Weaver J, Martin DP, Neff M, Stern EJ and Hudson LD: Incidence and outcomes of acute lung injury. N Engl J Med 353: 1685-1693, 2005.

2. Voigtsberger S, Lachmann RA, Leutert AC, Schläpfer M, Booy C, Reyes L, Urner M, Schild J, Schimmer RC and Beck-Schimmer B: Sevoflurane ameliorates gas exchange and attenuates lung damage in experimental lipopolysaccharide-induced lung injury. Anesthesiology 111: 1238-1248, 2009.

3. Winter J, Jung S, Keller S, Gregory RI and Diederichs S: Many roads to maturity: microRNA biogenesis pathways and their regulation. Nat Cell Biol 11: 228-234, 2009.

4. Dai R and Ahmed SA: MicroRNA, a new paradigm for understanding immunoregulation, inflammation and autoimmune diseases. Transl Res 157: 163-179, 2011.

5. Bartel DP: MicroRNAs: Target recognition and regulatory functions. Cell 136: 215-233, 2009

6. Tanaka S, Ishikawa M, Arai M, Genda Y and Sakamoto A: Changes in microRNA expression in rat lungs caused by sevoflurane anesthesia: A TaqMan ${ }^{\circledR}$ low-density array study. Biomed Res 33: 255-263, 2012.

7. Ishikawa M, Tanaka S, Arai M, Genda Y and Sakamoto A: Differences in microRNA changes of healthy rat liver between sevoflurane and propofol anesthesia. Anesthesiology 117 : $1245-1252,2012$

8. Liew FY, Xu D, Brint EK and O'Neill LA: Negative regulation of toll-like receptor-mediated immune responses. Nat Rev Immunol 5: 446-458, 2005.

9. Taganov KD, Boldin MP, Chang KJ and Baltimore D: NF-kappaB-dependent induction of microRNA miR-146, an inhibitor targeted to signaling proteins of innate immune responses. Proc Natl Acad Sci USA 103: 12481-12486, 2006.

10. Bazzoni F, Rossato M, Fabbri M, Gaudiosi D, Mirolo M, Mori L, Tamassia N, Mantovani A, Cassatella MA and Locati M: Induction and regulatory function of miR-9 in human monocytes and neutrophils exposed to proinflammatory signals. Proc Natl Acad Sci USA 106: 5282-5287, 2009.

11. O'Connell RM, Taganov KD, Boldin MP, Cheng G and Baltimore D: MicroRNA-155 is induced during the macrophage inflammatory response. Proc Natl Acad Sci USA 104: 1604-1609, 2007.

12. Moschos SA, Williams AE, Perry MM, Birrell MA, Belvisi MG and Lindsay MA: Expression profiling in vivo demonstrates rapid changes in lung microRNA levels following lipopolysaccharide-induced inflammation but not in the anti-inflammatory action of glucocorticoids. BMC Genomics 8: 240, 2007.

13. Tili E, Michaille JJ, Cimino A, et al: Modulation of miR-155 and miR-125b levels following lipopolysaccharide/TNF- $\alpha$ stimulation and their possible roles in regulating the response to endotoxin shock. J Immunol 179: 5082-5089, 2007.

14. Nahid MA, Satoh M and Chan EK: Mechanistic role of microRNA-146a in endotoxin-induced differential cross-regulation of TLR signaling. J Immunol 186: 1723-1734, 2011.

15. Riess ML, Stowe DF and Warltier DC: Cardiac pharmacological preconditioning with volatile anesthetics: From bench to bedside. Am J Physiol Heart Circ Physiol 286: H1603-H1607, 2004.

16. Tanaka K, Ludwig LM, Kersten JR, Pagel PS and Warltier DC: Mechanisms of cardioprotection by volatile anesthetics. Anesthesiology 100: 707-721, 2004. 
17. Liu R, Ishibe Y and Ueda M: Isoflurane-sevoflurane adminstration before ischemia attenuates ischemia-reperfusion-induced injury in isolated rat lungs. Anesthesiology 92: 833-840, 2000.

18. Lee HT, Ota-Setlik A, Fu Y, Nasr SH and Emala CW: Differential protective effects of volatile anesthetics against renal ischemia-reperfusion injury in vivo. Anesthesiology 101: 1313-1324, 2004.

19. Beck-Schimmer B, Breitenstein S, Urech S, De Conno E Wittlinger M, Puhan M, Jochum W, Spahn DR, Graf R and Clavien PA: A randomized controlled trial on pharmacological preconditioning in liver surgery using a volatile anesthetic. Ann Surg 248: 909-918, 2008.
20. Reutershan J, Chang D, Hayes JK and Ley K: Protective effects of isoflurane pretreatment in endotoxin-induced lung injury. Anesthesiology 104: 511-517, 2006.

21. Hofstetter C, Boost KA, Flondor M, Basagan-Mogol E, Betz C, Homann M, Muhl H, Pfeilschifter J and Zwissler B: Anti-inflammatory effects of sevoflurane and mild hypothermia in endotoxemic rats. Acta Anaesthesiol Scand 51: 893-899, 2007. 